\title{
Biomarkers associated with changes in bone mass of people living with HIV: a systematic review protocol
}

\begin{abstract}
Background: The introduction of highly active antiretroviral therapy strongly exposes individuals to the complications of chronification of the infection, like changes in mineral and bone metabolism. Measurement of bone mass is the most used static method to reflect changes. However, biochemical markers are capable of dynamically reflecting the steps in the process of bone component alterations, since the biomarkers of bone resorption and formation are released during the bone remodeling process. This systematic review
\end{abstract}

Aims: To identify of the possible markers and predictors of early alteration (s) in BMD and help in the management of future complications such as osteoporosis future.

Methods: The systematic review will search for literature, mainly observational (cohort and case-control) studies, with human participants aged between 18 and 65 years old. The exposures are various bone biomarkers. The outcome is low bone diagnosed by dual-energy X-ray absorptiometry (DXA) associated in group with laboratory-confirmed HIV/AIDS infection. There not the comparison group. Evidence will be identified from electronic searches of online databases of Medline, LILACS, Scopus, Google Scholar and Brazilian Digital Library of Thesis and Dissertations. Two reviewers will be involved in quality assessment and data extraction of the review. A data extraction form will be developed to collate data from selected studies. A checklist for quality assessment will be Cochrane Collaboration Tool to Assess Bias Risk for randomized clinical trials and Newcastle-Ottawa Scale for control cases and cohorts.

Discussion and Conclusion: This systematic review aims to systematically identify and summarise evidence on profile of bone biomarkers of HIV-infected adults which could be used in the prognostic model for early detection of low mass in PLWHA.
Volume 5 Issue 2 - 2018

\author{
Nathalia Sernizon Guimaraes \\ Federal University of Minas Gerais, Brazil
}

Correspondence: Nathalia Sernizon Guimaraes, Federal University of Minas Gerais, Rua SÃ $€$ Juliẽ $€ o$, Brazil, Tel 3197772844,Email nasernizon@hotmail.com

Received: February 21, 2018| Published: April 25, 2018

Keywords: biomarkers, bone and bones, antiretroviral therapies, early diagnosis, human immunodeficiency virus

Abbreviations: ART, antiretroviral therapy; BMD, bone mineral density; BMI, body mass index; DXA, dual-energy X-ray absorptiometry; HAART, highly active antiretroviral therapy; HIV, human immunodeficiency virus; PLWHA, people living with HIV/ AIDS; PRISMA-P 2015, preferred reporting items for systematic reviews and meta-analyses for protocols 2015

\section{Introduction}

Initially restricted to regional endemic areas and specific populations, Human Immunodeficiency Virus (HIV) infection spreads to alarming proportions without distinction of risk groups. ${ }^{1,2}$ Considered to be one of the major public health problems in low- or middle-income countries, approximately 5,754 individuals become infected every day worldwide (3). Of these, $90.7 \%$ are adults and $54 \%$ do not receive antiretroviral drugs (ARVs). ${ }^{3}$ It is also estimated that $40 \%$ or 14 million people are unaware that they are infected with the HIV. ${ }^{4,5}$

HIV is a retrovirus belonging to the Retroviridae family and Lentivirus genus, which has a high affinity for the receptor present on the surface of T-CD4+lymphocytes and macrophages. ${ }^{6}$ Upon invading the target cell of the infection, HIV replicates by installing a panel of selective depletion of T helper or T-CD4+lymphocytes and thus, the immune deterioration or immunosuppression of the host. ${ }^{6}$ During the first years of the epidemic, patients progressed to a complete state of immune deterioration, characterized by the presence of opportunistic infections and involuntary weight loss $(\geq 10 \%$ of usual weight), with a direct effect on the worsening of the clinical picture and an exponential increase in mortality of these individuals. ${ }^{7}$ To address this reality, clinical researchers have introduced ARV drugs into viral load control therapy and consequently the degree of immunosuppression of people living with HIV/AIDS (PLWHA). ${ }^{8}$

The introduction of highly active antiretroviral therapy (HAART) strongly exposes individuals to the complications of chronification of the infection, thus contributing to the appearance of metabolic and morphological changes. ${ }^{9-11}$ In addition to the increase in body weight, total cholesterol, triglycerides, LDL-cholesterol, as well as the reduction of HDL-cholesterol, insulin resistance and lactic acidosis, currently changes in mineral and bone metabolism have been observed as an event associated with continuous use of ARVs. ${ }^{12}$

Among the alterations in bone metabolism, previous studies indicated the loss of bone mass, especially the reduction of bone mineral density (BMD) and increased rates of fractures, among HIVinfectes compared with HIV-uninfected. ${ }^{13,14}$ Randomized clinical trials, that approached anti-retroviral naives patients have show that 
initiation of HAART'first year is associated with decrease in BMD of $2-6 \%$ in the spine and hip. ${ }^{15-18}$

The pathophysiology of the reduction of BMD is a multifactorial, with hypogonadism, smoking, alcoholism, low body mass index (BMI), imbalance of nutrients in the diet, vitamin D deficiency, and decreased organic capacity of bone tunnor. ${ }^{19}$ In comparison to the population without HIV infection, in PLWHA there is an imbalance in the process of bone remodeling. ${ }^{20}$ This is a dynamic and constant characterized by the alternation between matrix formation and bone resorption, allowing worn or damaged tissues to be renewed and replaced by new and healthy tissues, besides allowing the active participation in the mineral homeostasis of the organism, controlling the levels Serum calcium and phosphorus. ${ }^{19,20}$

Measurement of bone mass is the most used static method to reflect changes. ${ }^{21}$ However, biochemical markers are capable of dynamically reflecting the steps in the process of bone component alterations, since the biomarkers of bone resorption and formation are released during the bone remodeling process. ${ }^{22}$ In addition to acting as predictors of the rate of bone loss and consequently the risk of fracture, bone biomarkers may be protagonists in the selection of patients for ART alternation, monitoring the adherence and effectiveness of drug treatment. ${ }^{22}$

The physiology, clinical applications, analytical variability as well as possible ways to optimize analytical techniques of these biomarkers (enzymes, matrix proteins and collagen degradation products) have been extensively studied, believing that bone markers can complement the results of BMD in the management of future complications, such as osteoporosis. $^{21,22}$

A systematic review is therefore necessary to identify the association between bone biomarkers and low bone mass which might be associated with HIV infection. The results of the analysis can help in the identification of the possible markers and predictors of early alteration (s) in BMD and help in the management of future complications such as osteoporosis. This systematic review will identify, critically evaluate and interpret available evidence related to the clinical and laboratory characteristics associated with HIV/AIDS infection in adults in the world.

\section{Methods}

The steps of this systematic review will include defining the inclusion and exclusion criteria, finding studies, selecting those studies that address the review question and meet the criteria, and extracting and compiling data. This protocol adheres to the requirements of Preferred Reporting Items for Systematic Reviews and Metaanalyses Protocol (PRISMA-P). ${ }^{23,24}$ The review will conform to the requirements of the PRISMA.

\section{Research question}

This systematic review aims to systematically identify and summarise evidence on profile of bone biomarkers of HIV-infected adults which could be used in the prognostic model for early detection of low mass in PLWHA. Individual factors in studies with multivariable models of predictors of HIV infection will also be identified.

The review question is:

\section{What is the profile of bone markers associated with changes in bone mass of PLWHA?}

Population, exposure and outcome: This systematic review will consider only studies with human participants aged 18 years until 65 years old. The exposures are biomarkers of formation, reabsorption and regulators of bone metabolism. This biomarkers can be enzymes (ALP-bone), protein (P1NP, N1NP, osteocalcin) and collagen degradation' products (NTX, CTX). The outcome is low bone diagnosed by dual-energy X-ray absorptiometry (DXA) associated in group with laboratory-confirmed HIV/AIDS infection.

Study design: The types of studies most suitable for this aetiological review should be mainly observational (analytical) studies that compared groups and produced odds ratios or predictive values or likelihood ratios (case-control and cohort, both retrospective and prospective studies). However, experimental studies will not also be considered.

Search strategy: We will search the following electronic bibliographic databases: Medline, LILACS, Scopus, Google Scholar and Brazilian Digital Library of Thesis and Dissertations. The search strategy will include only related terms or describing the intervention. The search terms will be adapted for use with other bibliographic databases in combination with specific database filters for controlled trials, where available. Searches were performed between March and April, 2017. There will be no language restrictions. Studies published in the last five years (2012-2017) will be searched. Searches will be reperformed immediately prior to final reviews and additional studies retrieved for inclusion.

Inclusion/exclusion criteria: Inclusion criteria reflect those noted above for participants/populations, as well as the following: (a) crosssectional, case-control, cohort design, clinical trials, diagnostics studies, short communication; (b) that were performed with both male and female with HIV, aged between 18 and 65 years; (c) that assessed biomarkers associated with boné metabolism in patients with HIV; (d) that assessed bone mass by dual energy x-ray absorptiometry Exclusion criteria: (a) duplicate publications; (b) studies conducted with children, adolescents, elderly individuals; (c) studies conducted with pregnant or breastfeeding women; (d) review studies or case report; (e) experimental studies with animals; (f) research whose thematic was not in accordance with the objective of this review; (g) studies that assessed the PLWHA by comorbidities or by therapeutic intervention other than antirretroviral therapy; (h) Without evaluation of biomarkers or without the examination of bone densitometry.

Data analysis and synthesis: Selection process will involve the inclusion of potentially relevant publications and the removal of duplicates identified by reading the title and abstract, and those studies that did not meet the inclusion criteria. The full articles will be checked for eligibility by a comprehensive reading, and inclusion decision will be taken. After reading the full articles the final selection will be made. The process of extracting data will be carried out by two researchers and a third researcher will resolve any discrepancies. Data to be extracted are year of publication of studies, population characteristics, serological technique, outcomes. The analysis will adhere to the requirements of the PRISMA approach, and a PRISMA diagram will be included, showing the number of studies considered at each level of screening and assessment. 
Risk of bias (quality) assessment: For randomized clinical trials, we will use the Cochrane Collaboration Tool to Assess Bias Risk, for control cases and cohorts we will use the Newcastle-Ottawa Scale.

\section{Discussion}

The findings from the systematic review will be summarised as tabulations, and the strengths and weaknesses of the review will be discussed. Conclusions and recommendations on existing scientific evidence will be used to inform decision-making in the prediction model development. The patient characteristics will be tabulated according to the following categories 1 . Socio-economic (examples: sample, age, gender and antirretroviral use) 2. Type of study and sample calculation 3. Biomarkers used (training, reabsorption, hormonal regulators and inflammation) 4. Techniques used for the collection of biomarkers and low bone mass 5. Associations between bone biomarkers and changes in bone mass.

\section{Dissemination}

The systematic review will be submitted for publication. The findings of the systematic review will contribute to a postdoctoral fellow project and will be presented at conferences.

\section{Funding}

The research is not funded by any institution.

\section{Authors' contributions}

NSG wrote the protocol while AMK, MMG and UT critically appraised, edited and provided guidance during the development of the protocol. All authors approved the final version of the protocol and take responsibility for its content.

\section{Acknowledgements}

None.

\section{Conflict of interest}

The author declare that there is no conflict of interest

\section{References}

1. Faria RN, Rambaut A, Suchard MA, et al. The early spread and epidemic ignition of HIV-1 in human populations. Science. 2014;346(6205):56-61.

2. http://www.unaids.org/en/resources/documents/2015/GARPR_2015 guidelines

3. AIDS epidemic update. World Health Organization (WHO) \& UNAIDS Geneva; 2016.

4. Global AIDS up date 2016. World Health Organization (WHO). Geneva; 2016.

5. Review of data from People Living with HIV Stigma Index surveys conducted in more than 65 countries. UNAIDS. Geneva; 2016.

6. Korsman SNJ, Zyl GU, Nutt L, et al. Virologia. 1st ed. Rio de Janeiro: Elsevier; 2014.

7. Moore RD, Chaisson RE. Natural History of Opportunistic Disease in an HIV-Infected Urban Clinical Cohort. Ann Intern Med. 1996;124(7):633642 .

8. Dourado I, Veras MASM, Barreira D, et al. Tendências da epidemia de AIDS no Brasil após a terapia anti-retroviral. Rev Saúde Pública. 2006;40:
9-17.

9. Stanley TL, Grinspoon SK. Body composition and metabolic changes in HIV-infected patients. J Infect Dis. 2012;205(3):383-390.

10. Carr A, Emery S, Law M, et al. An objective case definition of lipodystrophy in HIV-infected adults: a case-control study. Lancet. 2003;361(9359):726735 .

11. Kibirige D, Ssekitoleko R. Endocrine and metabolic abnormalities among HIV-infected patients: A current review. Int J STD AIDS. 2013;24(8):603611.

12. Hileman CO, EckardAR, McComsey GA. Bone loss in HIV: a contemporary review. Curr Opin Endocrinol Diabetes Obes. 2015;22(6):446-451.

13. Brown TT, Qaqish RB. Antiretroviral therapy and the prevalence of osteopenia and osteoporosis: a meta-analysis review. AIDS. 2006;20(1):2165-2174.

14. Young B, Dao CN, Buchacz K, et al. Increased rates of bone fracture among HIV-infected persons in the HIV Outpatient Study (HOPS) compared with the US general population, 2000-2006. Clin Infect Dis. 2011;52(2):1061-1068.

15. Duvivier C, Kolta S, Assoumou L, et al. Greater decrease in bone mineral density with protease inhibitor regimens compared with nonnucleoside reverse transcriptase inhibitor regimens in HIV-1 infected naive patients. AIDS. 2009;23(7):817-824.

16. Van Vonderen MG, Lips P, van Agtmael MA, et al. First line zidovudine/ lamivudine/lopinavir/ritonavir leads to greater bone loss compared to nevirapine/lopinavir/ritonavir. AIDS. 2009;23(11):1367-1376.

17. Hansen AB, Obel N, Nielsen $\mathrm{H}$, et al. Bone mineral density changes in protease inhibitor-sparing vs. nucleoside reverse transcriptase inhibitorsparing highly active antiretroviral therapy: data from a randomized trial. HIV Med. 2011;12(3):157-165.

18. McComsey GA, Kitch D, Daar ES, et al. Bone mineral density and fractures in antiretroviral-naive persons randomized to receive abacavir-lamivudine or tenofovir disoproxil fumarate-emtricitabine along with efavirenz or atazanavir-ritonavir: aids clinical trials group A5224s, a substudy of ACTG A5202. J Infect Dis. 2011;203(12):1791-1801.

19. Harris W, Brown TT. Bone loss in the HIV-infected patient: evidence, clinical implications and treatment strategies. J Infect Dis. 2012;205(3):391-398.

20. Stellbrink HJ, Orkin C, Arribas JR, et al. Comparison of changes in bone density and turnover with abacavir-lamivudine versus tenofoviremtricitabine in HIV-infected adults: 48-week results from the ASSERT study. Clin Infect Dis. 2010;51(8):963-972.

21. Bone Mass Measurement: What the Numbers Mean. National Institutes of Health Osteoporosis and Related Bone Diseases. The U.S. Department of Health and Human Services: USA; 2015.

22. Hlaing TT, Compston JE. Biochemical markers of bone turnover - uses and limitations. Ann Clin Biochem. 2014;51(2):189-202.

23. Moher D, Shamseer L, Clarke M, et al. Preferred reporting items for systematic review and meta-analysis protocols (PRISMA-P) 2015 statement. Syst Rev. 2015;4:1.

24. Moher D, Liberati A, Tetzlaff $\mathrm{J}$, et al. Preferred reporting items for systematic reviews and meta-analyses: the PRISMA statement. Ann Intern Med. 2009;151(4):264-269. 